\section{PSICOLOGÍA DEL SUJETO CREATIVO/INNOVADOR $Y$ LAS NUEVAS FORMAS DE VIDA Y REPRODUCCIÓN}

\author{
Mikel Villarreal Sáez \\ Departamento de Psicología Social \\ Universidad del País Vasco
}

\section{PSYCHOLOGY OF THE CREATIVE SUBJECT/INNOVATOR AND THE NEW FORMS OF LIFE AND REPRODUCTION}

\begin{abstract}
Modern psychology has generated a great effort and huge research resources on the field of creative/innovative personality. The positivist epistemological and methodological research frameworks have been the predominant line on research methodology. All those resources and research commitment have not obtained yet any conclusive results and even less satisfactory results. The combination of creativity-innovation and productivity from the dominant psychological-managerial discourses set the direction of the predominant research. The various meanings that connote the terms "Information Society" and "Knowledge Society" entail each one of them separately different meanings and implications in relation to the innovative action of consumers and users.
\end{abstract}

KEY WORDS: Innovation; creativity; knowledge society; information Society.

\section{INTRODUCCIÓN}

La innovación y la creatividad se han convertido en valores específicos de nuestro tiempo. Además de un individualismo constriñente, los individuos de nuestro tiempo sentimos en nuestro entorno un marco de compulsión a la innovación, con un tipo de presión en el desempeño eficiente, en la disponibilidad para adaptarse a nuevas situaciones y necesidades, en la flexibilidad para realizar varios tipos de tareas. A la entusiasta valoración de lo nuevo y de lo novedoso por el mero hecho de serlo, le acompaña una devaluación de lo estable, de lo permanente, de lo sustancial. En esta edad de la creatividad y de la innovación abundan un tipo de industrias en las que encontramos expertos de todos los campos. De entre toda esta variedad de gentes que trabajan en la industria de la innovación y la creatividad sobresalen dos tipos de expertos: los psicólogos (que hablan de y para el sujeto individual), por un lado, y los directivos, gerentes, managers de los aparatos económicos
RESUMEN: La Psicología moderna ha generado un gran esfuerzo y enormes recursos de investigación en torno a la personalidad creativo/innovadora. Marcos epistemológicos y metodológicos positivistas experimentales han marcado el sentido predominante de de la investigación. Tantos recursos y dedicación investigadora no han obtenido hasta la fecha resultados concluyentes y mucho menos satisfactorios. La fusión entre creatividad-innovación y productividad desde los discursos hegemónicos psicológico-manageriales marcan el sentido de la investigación predominante. Los diferentes significados que connotan a los términos "Sociedad de la Información" y "Sociedad del Conocimiento" conllevan cada uno por su lado diferentes significados e implicaciones en relación con la acción innovadora de los consumidores y usuarios.

PALABRAS CLAVE: Innovación; creatividad; sociedad del conocimiento; sociedad de la información.

(que hablan desde y para la productividad), por otro. De esa manera surge, por mera hibridación, un tipo de Psicología "Managerial" con un discurso experto en todo lo relacionado con la moderna "tecné" de los procesos creativos e innovadores. En este Mundo Nuevo de la Sociedad de la Innovación y de la Creatividad, éstas se han constituido en una especie de imperativos morales para el sujeto de las sociedades tecnológicas contemporáneas.

\section{LA CREATIVIDAD, Y LA INNOVACIÓN: OBJETO DE ESTUDIO DE LA PSICOLOGÍA MODERNA}

Bien es cierto que la creatividad fue abordada en mayor o menor medida por grandes pensadores de la Psicología Moderna tales como Vigotsky, Piaget, Freud, o Maslow, entre otros. Sus obras no se hallan, sin embargo, en la corriente de la nueva Sociedad de la Innovación y la Creatividad. 
Desde los campos discursivos de una Psicología "Managerial" se escucha, de forma más audible, que uno de los factores llave que habria de conducir la civilización hacia adelante es instalar un nuevo impulso innovador y creativo en nuestra sociedad

Si tomamos los años 50, del pasado siglo, como el inicio de la consolidación de la investigación psicológica moderna (con el método experimental como protector de la esencia científica), fue en los años ochenta cuando un pequeño número de grandes cuestiones polarizaron la mayoría de este tipo de investigadores: - La Personalidad creativa, y las técnicas de pensamiento creativo.

Paralelamente, ya había emergido un campo disciplinar en psicología, una Psicología Comunitaria, orientada al desarrollo de estrategias de intervención para afrontar problemas sociales y con el marchamo de promotor el cambio social, y proponiendo literalmente una Psicología de la Innovación Social. El programa "Experimental Social Innovation and Dissemination", con esta vocación de intervención social cara a la reforma y mejora de los problemas sociales, se situó en su día como una de las vanguardias en la acción investigadora y en la promoción del cambio social en el ámbito profesional de los psicólogos comunitarios norteamericanos. Esta Psicología Comunitaria pronto echará anclas en las latitudes centro y suramericanas, y también en Europa (Fairweather et al., 1974; Tornatzky et al., 1980; Fairweather y Davidson, 1986). La Psicología Comunitaria tiene un lugar de influencia en la profesión psicológica, a pesar del corto período de tiempo en que se ha desarrollado. Nociones tales como prevención y "empoderamiento" (Empowerment), hoy ya de uso común, se desarrollaron en esta corriente de psicólogos comunitarios (Hazel y Onaga, 2003) quienes, ya en la época de los años 70 del siglo XX, estaban utilizando la noción de Innovación Social con vistas a reformar y enfrentarse a los problemas sociales.

Pero en la investigación de la psicología del sujeto innovador y creativo, en los años 90 del siglo XX, se asiste a una explosión de tópicos y de perspectivas teóricas y metodológicas en el estudio de la creatividad; una situación en constante expansión de una variedad de enfoques y de marcos teóricos y metodológicos, pero no epistemológicos. En este mismo y extenso campo de investigación se tiene la impresión de que los investigadores de un subcampo ignoran y desconocen los avances en el otro campo. Los que trabajan en el nivel de análisis individual no saben qué resultados obtienen los que investigan en un nivel de inserción grupal. Los que estudian en el nivel organizacional apenas miran lo que se hace en los otros dos niveles de análisis mencionados. Los hay que defienden la imperiosa necesidad de desarrollar el nivel de Análisis Institucional de la Innovación (Holligsworth, 2000; Amabile y Kramer, 2002).

Pero lo que ocurre es que, en la mayoría de los casos, la investigación sobre la creatividad o la innovación se da en un sólo nivel de análisis, sin intentar siquiera armonizar o superar el reduccionismo que implicaría cada nivel de análisis.

Plucker y Runcon (1998) reconocen de alguna manera un cierto sentimiento de fracaso en lo relativo a la definición y a la medición de la creatividad. $Y$, de hecho, ya entrados en el siglo XXI, se sigue discutiendo y debatiendo sobre la definición de creatividad, sin que podamos observar mínimos consensos al respecto.

Por otra parte, las cuestiones de definición y de operacionalización empleados en los paradigmas de investigación experimentales, han llegado a ser progresivamente complejos, de forma que, finalmente, la capacidad para definir con precisión (tal y como se exige en el quehacer "científico") qué es lo que se entiende por creatividad permanece justamente estancada (Kaufman, 2003 y 2007).

\section{LA INNOVACIÓN Y LA CREATIVIDAD DEL SUJETO "PSICOLÓGICO" INDIVIDUALIZADO}

Como hemos apuntado anteriormente, la creatividad de las personas ha sido el objeto de estudio típico de los enfoques que desarrollan los paradigmas experimentales en Psicología, aunque también se ha apoyado en el estudio de casos, o en investigaciones a partir de técnicas de cuestionario que operacionalizan la creatividad bajo la perspectiva de ser algo esencialmente duradero, como un conjunto de rasgos de personalidad (de un sujeto psicológico) que tendrian una manifiesta estabilidad en el tiempo. El "Torrance Test of Creativity Thinking" es un 
buen ejemplo de la posición teórico-metodológica de este género (Torrance 1966/1974).

Por su parte, la noción de creatividad que defiende Kirton (1994) se centra alrededor de procesos cognitivos de generación de "novedad" y de "resolución". El autor de la obra "Adaptors and Innovadors. Styles of Creativity and Problem Solving", admite que es "en una época reciente cuando la noción de innovación ha llegado a ser sinónimo de progreso tecnológico, y que se ha constituido como uno de los criterios de creatividad" (Kirton, 1994, p. xxxi). Los estilos cognitivos "Adaptation-Innovation", son vistos aquí como una dimensión de los procesos cognitivos, y se asume que éstos, en general, están basados en preferencias individuales en la forma de organizar y procesar la información. Se los considera como diferencias individuales consistentes, $y$, de alguna manera, pertenecientes a la propia naturaleza de los individuos, en la medida en que los estilos "adaptators-innovators" se desarrollarían alrededor de rasgos subyacentes de personalidad (Kirton, 1994).

La noción de Estilos de Pensamiento ha sido empleada en el denominado "paradigma de la Innovación, Conocimiento y Aprendizaje", considerando la hipótesis del "cerebro creativo" -"creative brain"-. El trabajo titulado "Thinking Styles of technical knoledge workers in the System of innovation paradigma" (Amadi-Echendu, 2006), constituye un buen ejemplo de cómo escudriñar dentro del cerebro y tratar de encontrar su correlato funcional respecto al pensamiento creativo, y sus resultados, de acuerdo con los presupuestos teórico-metodológicos de este estudio, indicarían una preferencia por el estilo de pensamiento cerebral derecho, como un tipo de "mentalidad cerebral", que serian representativos del comportamiento empresarial.

La literatura científica, que intenta relacionar los procesos cognitivos y los componentes de un tipo de comportamiento creativo, presenta un cuadro muy sombrío, que es reconocido por los propios investigadores en este campo y defensores de los presupuestos epistemológicos y metodológicos mencionados. Así, Mumford y Antes (2007), después de realizar un cuadro del estado de la cuestión, Ilaman, en este sentido, a ser muy cautos ante la pretensión de basar los logros creativos en un modelo simple de un tipo o estilo de conocimiento determinado o de supuestos procesos cognitivos implicados.
De entre las cuestiones en que se ha detenido este gran campo de investigación tenemos que la relación entre creatividad e inteligencia estarian modestamente relacionados (Sivia 2008). Otros estudios subrayan el papel central de la curiosidad y la confianza en sí mismo y la creatividad (Kashdan y Finchan, 2002; Kaufman, 2002). Se ha estudiado, también, la dimensión emocional y la creatividad (Vosburg, 1998) sin que se hayan obtenido conclusiones contundentes (Clapham, 2001; Amabile et al., 2005). Desde los estudios de casos que se vierten en la revista "American Psychologist" (April, 2001), se puede constatar cuán difícil resulta intentar identificar variables esenciales para la creatividad basadas en diferencias individuales.

Nuevas aproximaciones sobre el comportamiento creativo y sobre los procesos creativos ponen su atención en el "desempeño" de las personas en grupo y su relación con una mayor rendimiento y capacidad creativa. Se trata de comparar el desempeño (rendimiento) o la solución de problemas de las personas en solitario, o en grupo (Svensson et al., 2002; Treffinger et al., 2006). Las conclusiones de este tipo de investigación llevan a diferentes resultados, los cuales pueden estar en gran parte inducidos por el tipo de tareas experimentales, y por la propia conceptualización y metodología de investigación empleados. Se plantea, en la misma línea argumental, que los problemas solucionados creativamente varían considerablemente en su complejidad, en las bases de conocimientos requeridos, y en la cantidad de pensamiento convergente o divergente que se precisa.

Brophy (1998) al proponer el modelo "Tri Level Maching Theory", hace hincapié en el hecho de que un proceso de solución de problemas complejo conlleva la activación tanto de un pensamiento convergente como de un pensamiento divergente en continua alternancia (Brophy, 2006). La importancia de provocar un pensamiento divergente a gran escala para el cambio social se encuentra destacada en los sobresalientes trabajos de Moscovici sobre la influencia de las minorias activas (Moscovici, 1997; Mugny Movimientos Sociales). Las minorias activas, movimientos sociales exitosos, a través de la activación de los procesos de validación, inducirían en la población, blanco de su influencia, a un tipo de pensamiento divergente, como un primer paso de los distintos procesos de la llamada influencia minoritaria. Sin la acción innovadora de estas 
minorias, no podriamos comprender en su amplitud el cambio social y político.

Paulus y Yang (2002) sostienen que los grupos que comparten ideas acabarian por llegar a ser más productivos. Un estudio cualitativo en el mundo empresarial identifica las siguientes conductas relacionadas con lo que denominan "momentos de creatividad colectiva: ayuda mutua en la búsqueda; ayuda mutua en la entrega; reenmarcamiento reflexivo; reforzamiento" (Hargadon y Bechky, 2006).

Otro de los aspectos en que se han detenido algunos investigadores es en la cuestión de si una mayor o menor diversidad, o una mayor o menor homogeneidad, en el seno de grupos de trabajo conduce a resultados más positivos o más negativos (Mannix y Neale, 2005). La diversidad tendería a mejorar el desempeño de las tareas creativas en grupos (Hennessey y Amabile, 2010).

Se ha puesto énfasis, también, en la motivación como un factor relacionado con la creatividad. A este respecto la motivación intrínseca, es decir, el impulso a hacer algo por el propio interés del sujeto, o porque se divierte o le satisface de alguna manera, sería más inductiva para la creatividad que la motivación extrínseca (Hennessey, 2003). Asimismo, Farmer et al. (2003) estudiaron cómo la creatividad de los individuos era más alta cuando ellos mismos se percibían como empleados creativos, y cuando percibian que sus organizaciones valoraban su trabajo creativo.

En conclusión, muchos de los investigadores de lo que denominariamos un campo de investigación psicológica sobre la creatividad y la innovación reconocen que tanto esfuerzo investigador ha dado resultados muy atomizados, muy ligados a un reductivismo propio del nivel de análisis en que la investigación se enmarca. Hay un progresivo consenso sobre la necesidad de estudiar estos procesos creativos de forma más amplia y multidisciplinar. Pero la práctica sigue siendo reduccionista, y por lo tanto muy pobre en cuando a las posibilidades explicativas. Se necesitan, se proclama en estos mismo círculos, modelos y metáforas más amplios, más holísticos; marcos que abandonen perspectivas de investigación lineal y que incluyan mayores niveles de complejidad (Hennessey y Amabile, 2010).
Mientras tanto, la reflexión acerca de la creatividad, del sujeto creativo, de los estilos creativos o incluso de las organizaciones creativas seguirá siendo un producto de baja calidad destinado a satisfacer demandas urgentes, sobre todo demandas del mundo empresarial "psicologizado" que percibe la creatividad del individuo psicologizado como factor clave de la innovación, de la motivación laboral y de la productividad.

En un contexto de modernidad avanzada y capitalismo del valor añadido, el discurso de la empresa (y en particular el discurso del Management) se ha culturizado hasta el punto de arrebatar al "Mundo de la Cultura" su lenguaje y sus objetivos manifiestos. La creatividad, característica psicológica del artista, se ha convertido en una demanda empresarial.

La productividad y la creatividad se habian presentado como conceptos antitéticos dentro de una misma tradición epistemológica y psicológica. El sujeto productivo pertenece al ethos burgués y a su correlativa empresa tecno-científica de dominio del mundo. Empeño, inversión, sacrificio, trabajo, disposición, severidad, etc., son los términos en los que se expresa un sujeto que se ha dispuesto a producir, a regular el mundo, y a sí mismo. No pretende ser un pequeño dios que crea en sentido radical, pero sí la figura que propuso el humanismo renacentista y que inauguró la Modernidad: un sujeto, el individuo, dispuesto por su naturaleza a regular el mundo, y muy en particular su mundo histórico y social.

La lógica de la acumulación se ha podido desarrollar como una tendencia hacia el infinito gracias a la abstracción del valor, el capital, pero también gracias al sujeto psicológico propio de las relaciones del capital. Fundamentalmente un sujeto movido por una fuerza interna propia (su psique), un sujeto equivalente en su estado inicial y que debía adquirir un valor diferencial en su desarrollo, siempre entendido como responsabilidad propia.

A diferencia del sujeto productivo, el sujeto creativo si no era un pequeño dios, sí se le concedía un genio interior. La creatividad se refugió en el mundo artístico, precisamente como mundo contrapuesto a lo cotidiano, a lo práctico, a lo productivo. La imagen del genio creador del neoplatonismo renacentista, de Ficino muy en especial, dio lugar a la imagen del artista inspirado y genial. La creación genial 
se originaba en impulsos irracionales, puramente creativos, sensibles y corporales, aunque el establecimiento de cánones y academias fue despojando de genialidad al arte, para hacerlo precisamente más productivo y regular.

La imagen del artista afectó también al científico, sobre todo al científico especulativo. Creadores desinteresados y modernos, las figuras del artista y el científico serian incorporadas y representadas por los protagonistas de dos campos de creciente autonomía, el campo artístico y el campo científico; campos que han acumulado el capital simbólico de la innovación y la invención, valores capitales de la Modernidad (Romo, 2007).

La ciencia hubo de oscilar entre la observación teórica y la creatividad entendida como facultad computacional, por un lado, y la aplicación práctica y una creatividad entendida como desbordamiento y divergencia cognitiva, por otro.

La Modernidad se había desarrollado de tal manera que estas líneas habían sido siempre más complementarias que opuestas. Pero esa complementariedad se ha convertido en fuerte unión en la alta modernidad. Para entender el porqué y el cómo de esa unión habría que atender a la división social del trabajo creativo o, si se quiere, simplemente, a la división social de la creatividad.

Antropológicamente hablando, no hay grupo ni estrato social carente de creatividad. Pero tampoco grupo social en el que no se produzca una división del hacer creativo. Por un lado estarían las innovaciones cotidianas -aquéllas de carácter práctico y empírico, basadas en la experiencia informal-, y por otro, las innovaciones extraordinarias -más especulativas y teoréticas, en las que predomina la investigación o búsqueda formalizada de dichas innovaciones-. Hasta el siglo diecinueve fue creciendo la diferenciación entre ambas formas de creatividad en las sociedades occidentales. Entonces el corte político del romanticismo inició la recuperación de la creatividad inmanente a las formas de vida. Se revalorizó lo popular y se inventó lo folklórico, hasta el punto de que la legitimidad del genio artístico (o incluso científico) se subordinó a la expresión del genio colectivo. El arte por el arte, que los burgueses productivos habian despreciado, giró para reencontrarse con la vida y consecuentemente con el sistema productivo. La creatividad se preparaba para aterrizar sobre ese siste- ma de productividad infinita. Las vanguardias prepararon el camino desde el arte a la producción industrial, pero a su vez el desarrollo tecno-científico, promovido sobre todo por necesidades bélicas, estaba abriendo la alta tecnología a la vida ordinaria de las masas populares. El automóvil, los ansiolíticos, el teléfono móvil o la televisión son buenos ejemplos, y en ellos se fusionan la productividad y la creatividad tanto en los objetos como en los sujetos. La obra abierta queda en manos de la creatividad del usuario. Cultura pop, cultura de masas, uso de la innovación tecnológica, modernismo, consumo de modas efímeras, etc., son las manifestaciones de ese encuentro.

No hay una psicología estricta del sujeto creador (moderno), ni del sujeto creativo (ultra o hipermoderno) porque la fusión histórica de productividad y creatividad ha desbordado al sujeto. Ni la psicología intelectualista de origen cartesiano, ni la psicología vitalista del genio irracional, pueden dar cuenta de la creatividad; la reducen a facultad o potencia individual actualizada según contextos, o bien a un cálculo computacional o arte combinatorio, o bien a un instinto o tendencia hacia la divergencia cognitiva.

Hoy en día, la fantasía y otras facultades de la sensibilidad, la empatía, por ejemplo, se han situado al lado de la memoria, el razonamiento o la percepción. Conceptos como "inteligencia emocional" o "inteligencia creativa" pretenden casar términos que se pensaron como opuestos, y no lo hacen porque la psicología haya progresado en su entendimiento o en su descripción del ser humano. Lo hacen porque dan cuenta de las demandas de su contexto histórico. Un contexto en el que los campos autónomos de creatividad han sido abiertos y subsumidos bajo las formas de vida ordinarias del capitalismo global de consumo. Y en el que la subjetividad se ha convertido en una característica de los objetos y de las formas de consumo. Lo mismo creemos sobre la creatividad.

La psicología extraordinaria del genio creativo, de su inspiración y su pasión, se ha convertido en modo de producción porque lo que se produce es valor de diferencia, el valor añadido de lo distinto y lo distinguido. Lo que antes estaba destinado a unas pocas élites de refinado consumo (de gasto ostentoso), se ha convertido en el objeto del consumo de masas. De esa manera, la psicología de la creatividad también se ha trasladado de su atención al arte y al genio creativo, a las formas de vida y de producción. 
Sin embargo, no ha abandonado con la misma decisión la visión intelectualista de la creatividad. Así que, como hemos visto, sigue hablando de inteligencia creativa o de sujetos creativos.

No hay una psicología del creativo, ni una mente creativa per se, como no hay estilos creativos en abstracto fuera de las situaciones particulares de creación. El proceso creativo no se inicia en la psiqué de un individuo ordinario o extraordinario, ni en la conjunción de una serie de pautas o en la implementación de protocolos de actuación. El proceso creativo es precisamente la creación de objetos y sujetos, de situaciones y de organizaciones, que son 0 no creativas en tanto y en cuanto se apartan y se diferencian de aquellos y aquellas en las que y por las que han surgido.

\section{La inNOVACión SOCIAL EN las "Sociedades del Conocimiento" vs. la innovación tecnológica DE LA "SOCIEDAD DE LA INFORMACIÓN"}

Nuestra época contemporánea está asistiendo a profundas transformaciones que, en parte, están relacionadas con la introducción y generalización de la nuevas Tecnologías de Información y de la Comunicación, entre otras. De ahí que haya un sentimiento generalizado de que estamos en un profundo cambio de nuestras sociedades vinculado al impacto, difusión y generalización de dichas tecnologías.

Ya en los años 90 del siglo pasado se extendió el término "Sociedad de la Información y de la Comunicación", para caracterizar nuestra nueva época, y fue utilizado por todas las instancias fundamentales de los países más desarrollados. Los términos que usamos para definir nuestras sociedades no son neutros, los discursos que los envuelven no son desinteresados, sino que especialmente contienen una carga ideológica.

La Sociedad de la Información y de la Comunicación podría tomarse como una construcción política e ideológica que ha tenido éxito y legitimación en un período de ensanchamiento y apertura de un sistema capitalista hacia la globalización económica. Las nuevas tecnologías de la información y de la comunicación han desempeñado un papel clave en la aceleración de la globalización econó- mica, pilotada por los actores más representativos de una economía ultra o neoliberal. La Sociedad de la Información y de la Comunicación estaría, por tanto, muy relacionada con las innovaciones tecnológicas y con el papel político económico que desempeñaron a partir de las dos últimas décadas (Mattelart, 2001).

Por su parte, cuando usamos la noción de "Sociedades del Conocimiento", estariamos poniendo de relieve una dimensión de transformación social, cultural, económica, política e institucional. Hablar de Sociedades del Conocimiento nos permitiria abarcar mejor la complejidad y el dinamismo de los cambios que están teniendo lugar, en la medida en que el conocimiento es una cuestión crucial, no sólo para el crecimiento económico, sino también para el desarrollo de muchos más sectores de la sociedad (Khan, 2003; Ambrosi et al., 2005).

En la Sociedad de la Información y de la Comunicación no es evidente que quepamos todos, mientras que sí nos ofrece mayores posibilidades de inclusividad la Sociedad del Conocimiento, una sociedad o unas sociedades en las que todos puedan crear, consultar, utilizar y compartir la información y el conocimiento para potenciar y promover su desarrollo sostenible, mejorar la calidad de vida de las personas y desarrollar sus potencialidades (Mattelart, 2001).

Para este segundo enfoque, lo fundamental no estaría tanto en la información, cuando en la Sociedad, o en las sociedades. Mientras la Sociedad de la Información haría referencia a los datos, a las tecnologias de la transmisión y al espacio de almacenamiento, la perspectiva de la "Sociedad del Conocimiento" pone el foco de interés en los seres humanos, en las innovaciones que realizan las personas en el uso de los artefactos tecnológicos de formas no imaginadas por sus diseñadores, en las culturas y en nuevas formas de organización y de comunicación que surgen de "abajo-arriba".

Es decir, frente a esta visión tecnocéntrica de los discursos hegemónicos de la "Sociedad de la Información" en los que el conocimiento quedaría reducido a su función económica y en la que la innovación estaría promovida de "arriba-abajo" (Burch, 2006), quienes defienden el término "Sociedad del Conocimiento" estarían proyectando una visión más integral, y un conocimiento humano y para la 
humanidad, y, asimismo, reconociendo el papel que juega un tipo de innovación horizontal y de abajo-arriba.

Una "Sociedad del Conocimiento" en la que cabe partir del reconocimiento de la habilidad de los individuos para asumir su autonomía y su libertad, atiende mucho más a cómo muchos individuos llegan a constituirse en sujetos desplegando formas de autonomía en una amplia variedad de prácticas: de trucos y trampas, con sustituciones funcionales y apropiaciones ilegales, si cabe, implantando nuevos hábitos cotidianos, nuevos estilos de vida, etc. Los sujetos consumidores de la Sociedad de la Información serian, en la Sociedad del Conocimiento, usuarios capaces de inventar sus propias formas y prácticas enfrentadas al mundo impuesto por las industrias culturales y por las tecnologías de la Comunicación (Cardon, 2005).

Una comparación entre los términos de "Absorción Cognitiva" versus procesos de "Apropiación", puede ilustrar más en concreto los diferentes sentidos y connotaciones de los términos arriba mencionados: "Sociedad de la Información" versus Sociedad del Conocimiento".

El concepto de "Absorción cognitiva" (Agarwal y Karahana, 2000), se inscribe en el intento de explicar por qué los usuarios desarrollan conductas particulares respecto a las tecnologías de la Información, que están predominantemente diseñadas desde "a prioris" funcionales e instrumentales para la satisfacción de impulsos e intenciones de uso individual. Subyace en esta concepción un sujeto psicológico, cuyo sistema mental se representa como susceptible de ser absorbido por las potenciales del software, aspirado por "fuerzas" cada vez más perfeccionadas y poderosas del diseño de estas Nuevas Tecnologías. Un sujeto psicológico capaz también de absorber cognitivamente las fuerzas innovadoras que acompañan a la venida de las nuevas tecnologías de la Información y de la Comunicación.

La "Absorción Cognitiva", un constructo multidimensional que pone su acento en definirse como un forma de "profundo compromiso con el software", se exhibiría a través de varias dimensiones: disociación temporal, inmersión focalizada, elevado grado de disfrute (placer), control, curiosidad, utilidad percibida, facilidad percibida de uso, capacidad lúdica, y grado de innovación personal. Estas dimensiones mencionadas en el modelo de la Absorción Cognitiva no desarrollan una discontinuidad con la tradicional perspectiva de la psicología de los rasgos de personalidad. El constructo conceptual de la "absorción cognitiva", intentaría capturar la totalidad de una experiencia individual con el nuevo software, y pretendería desarrollarse y fundamentarse en las líneas maestras de la investigación de la psicología cognitiva y de una Psicología Social que pone en marcha unos marcos de investigación centrándose en la disección de la estructural mental y emocional de los individuos (Agarwal y Karahana, 2000).

En una dirección radicalmente diferente estarían las teorías de la "Apropiación", desde perspectivas que intentan desarrollar una teoría sociopolítica de los juegos de utilización y uso, poniendo la atención en los procesos progresivos de interiorización de las habilidades técnicas y cognitivas que despliegan los individuos y los grupos que usan dichas tecnologías para sus prácticas cotidianas.

La tecnología de "Apropiación Social" como perspectiva ofrece a las personas la capacidad y el poder de actuar, de adaptarse y de crear, aun cuando las mismas tecnologías prescriban formas propias de realización y de actuación.

El propio éxito de las nuevas tecnologías confirmaría que los "sujetos usuarios" son jugadores esenciales en los procesos de innovación.

\section{Conclusiones}

Así pues, en estas manifestaciones de creación cooperativa, de usos divergentes e innovadores, etc., es posible encontrar una psicología de la creatividad que vaya más allá del paradigma moderno del sujeto psicológico. Mantenemos "psicología" porque se refiere al comportamiento y sus bases significativas en este caso inmanentes a la interacción y a las relaciones; y mantenemos "creatividad" porque esos fenómenos son básicamente consecuencias no esperadas producidas sin intención, porque son apertura de nuevas formas de interacción y de cursos de acción no previstos. $Y$, en todo caso, porque suponen un cambio (no acumulativo, ni progresivo o tendencial, sin más) respecto a las condiciones en que se han desplegado. No hay individuo o sujeto individual creativo a priori, ni producto ni objeto "creativo" antes del proceso de configuración de ambos. Se producen en cuanto que participan en la red 
de interacción. Y que se produzca o no una novedad, una "creación", tampoco es predecible linealmente de dichas condiciones.

Esta psicología de la creatividad es post-psíquica, si por psiqué entendemos una instancia interna al sujeto; es fuertemente social e incluso adquiere rasgos de psicología colectiva, siempre que se tome a lo colectivo como relación, y no como sustancia o entidad psíquica. Quizás sea en el diseño de bienes de consumo, máquinas o muebles, donde esta psicología de la creatividad nos dé pistas de por donde avanzar. El uso se entiende en esas psicologías ergonómicas como interacción entre sujetos y objetos difusos en su "subjetividad" y "objetividad" y que disponen de diferentes grados de participación en la agencia del proceso (del uso) que es a su vez un proceso abierto (de transformación).

La fórmula "diseño emocional" de uno de los más conocidos autores de esta psicología del uso (Norman 2004) sintetiza la fusión de productividad y creatividad a la que aludíamos más arriba, y da cuenta de la extensión de la creatividad a las formas de vida ordinarias. El uso de los objetos cotidianos es una cuestión creativa no sólo para los diseñadores de los mismos; uno de los vectores fundamentales de su diseño es la usabilidad o apropiabilidad: -qué harán con ello los usuarios y cómo lo harán, -qué tipo de emociones se pondrán en juego. Sería estúpido y corto de miras entender esto como un mero feedback entre diseñador y usuario. El problema es que en la vida cotidiana en las denominadas formas de vida tecnológicas (Lash 2002), todos los objetos tienen carácter de interfaz y, por tanto, su uso es siempre un uso cognitivo y emocional indisolublemente, $y$, además, un uso abierto.

Sin embargo, conviene no exagerar el carácter creativo de las prácticas en las formas de vida tecnológicas. La tendencia de usuarios y diseñadores es la de ir cerrando y definiendo, en protocolos estrictos y especificaciones de diseño, lo que se puede y se debe hacer.

Confort cognitivo-emocional, por un lado, y deseo de retener un estatuto "artístico", por otro, se confabulan en la tendencia a asegurar y estabilizar formas recurrentes de interacción, sea entre seres humanos, o entre éstos y sus útiles.

Zonas de incertidumbre, aún abiertas o en proceso de apertura, sirven de refugio y de probeta de las propuestas creativas. Esas zonas suelen ser poco domésticas, problemáticas y conflictivas. Conflicto y creatividad se presentan de la mano, tal y como describieron Moscovici y sus colaboradores de las minorias activas y la influencia minoritaria (Moscovici, 1979; Mugny, 1981).

\section{BIBLIOGRAFÍA}

Agarwal, R. y Karahana, E. (2000): "Time flies when you are having fun: cognitve absortion and beliefs about information usage", MIS Quarterly, 24: 665-694.

Amabile, T. M.; Hadley, C. N. y Kramer, S. J. (2002): "Creativity under de gun", Harvard Bus. Rev., 80: 52-61.

Amabile, T. M.; Barsade, S. G.; Mueller, J. S. y Staw, B. M. (2005): "Affect and creativity at work", Admi. Sci. Q., 50: 367403.

Recibido: 16 de junio de 2010

Aceptado: 26 de octubre de 2010
Amadi-Echendu, J. E. (2007): "Thinking Styles of technical knowledge wor- kers in the systems of innovation paradigm", Technological Forecasting \& Social Change, 74: 1204-1214.

Ambrosi, A.; Peugeot, V. y Pimienta, D. (2005): Word Matters: multicultural perspectives on information societies, C \& F Éditions.

Brophy, D. R. (1998): "Understanding, measuring, and enhanding individual creative problem-solving efforts", Creativity Research Journal, 11: 123-150.

Brophy, D. R. (2006): "A comparison of individual and group effots to creatively solve contrasting types problems", Creativity Research Journal, 18: 293315. 
Burch, Sally (2004): Se cayó el sistema. Enredos de la sociedad de la información, Quito: ALAI.

Cardon, D. (2005): "Innovation by usage», en Ambrosi et al., World Matters: multicultural perspectives on information societies, C \& F Editions.

Clapham, M. M. (2001): "The effects of affect manipulation and information exposure on divergent thinking", Creatitiviy Research Journal, 13: 335-350.

Courrier, Y. (2003): Société de I'Information et technologies. http://www.unesco. org/webworld/point.

Fairweather, G. W.; Sanders, D. H. y Tornatzky, L. G. (1974): Creating change in mental health organization, New York: Pergamon Press.

Fairweather, G. W. y Davidson, W. S. (1986): Community experimentation, New York: McGraw-Hill.

Farmer, S. M.; Tierney, P. y Kung-McIntyre, K. (2003): "Employee creativity in Taiwan: an application of role identity theory", Academic. Management. J., 46: 618-630.

Hargadon, A. B. y Bechky, B. A. (2006): "When collections of creative become creative collectives: a field study of problem solving at work", Organ. Sci., 17: 484-500.

Hazel, K. L. y Onaga, E. (2003): "Experimental social innovation and dissemination: The promise and its delivery", American Journal of Community Psychology, v. 32, n. ${ }^{\text {s }}$ 3/4: 286-294.

Hennessey, B. A. y Amabile, T. (2010): "Creativity", Annual. Review. Psychol., 61: 569-598.

Holligsworth, J. R. (2000): "Doing Institutional analysis. Implications for the study of innovations", Review of Int. Pol. Econ., 7, 4: 595-644.
Kaschdan, T. B. y Fincham, F. D. (2002): "Facilitating creativity by regulating curiosity. Comment", American Psychologist., 57: 373-374.

Kaufman, G. (2002): "Creativity and confidence: price of achievement? Comment", American Psychologist., 57: 375-376.

Kaufman, G. (2003): "What to messure? A new look at the concept of creativity", Sandinavian Journal of Education Research, 47: 235-251.

Kaufman, J. C.; Lee, J. y Lee, S. (2007): "Captions, consistency, creativity, and the consensual assessment technique: new evidence of reliability", Thinking Skills Creativity, 2: 96-106.

Khan, A. W. (2003): "Towards Knowledge Societies. An Interview", World of Science, vol. 1, n. ${ }^{\circ} 4$.

Kirton, M. (1994): Adaptors and Innovators. Styles creativity and problem solving, London: Routledge.

Lash, S. (2002): Critique of Information, New York: Sage.

Lee, S. (2008): "Commentary: reliability and validity of uniqueness scoring in creativity assesment", Psychol. Aesthet. Creat. Arts, 2: 103-108.

Mannix, E. y Neale, M. (2005): "What differences make a difference? The promise and reality of diverse teams in organizations", Psychol. Public. Int., 6: 31-55.

Moscovici, S. (1979): La Psychologie des minorités actives, Paris: Presses Universitaires de France.

Mugny, G. (1981): El poder de las minorías. Psicología social de la influencia de las minorías e ilustración experimental, Barcelona: Ed. ROL.

Mattelart, A. (2001): Histoire de la Société de I'Information, Paris: Ed. La Decouverte.
Mumford, M. D. y Antes, A. L. (2007): "Debates about the 'general' picture: cognition and creative achievement", Creativity Research Journal, 19: 367374.

Paulaus, P. B. y Yang, H. C. (2000): "Idea generation in groups: a basis for creativity in organizations", Organiz. Behav. Hum. Decis. Process, 82: 7687.

Plucker, J. A. y Runco, M. A. (1998): "The death of creativity measurement has been greatly exaggerated: current issues, recent advances and future directions in creativity assessment", Roeper Rev., 21: 36-3.

Romo, M. (2007): "Psicología de la ciencia y de la creatividad", Revista Creatividad y Sociedad, n. ${ }^{\circ}$ 10: 7-31. www. creatividadysociedad.com.

Silvia, P. L. (2008): "Creativity and intelligence revised: a latent variable analysis on Wallach and Kogan, 1965", Creat. Res. J., 20: 34-39.

Svenson, N.; Norlander, T. y Archer, T. (2002): "Effects of individual performance versus group performance with and without de Bono techniques for enhance creativity", Korean Journal Problem-Solving, 10: 4959.

Torrance, E. P. (1966/1974): Torrance, test of creativity thinking norms - Technical manual, Lexington, MA: Ginn.

Treffinger, D. J.; Isaken, S. G. y Dorval, K. B. (2006): Creative Problem Solving: an introduction, Waco, TX: Prufrock.

Vosburg, S. K. (1998): "Mood and the quantity and quality of ideas", Creativity Research Journal, 11: 15-31.

Weshler, S. (2006): "Validity of the Torrance test of creativity thinking to the Brazilian culture", Creativity Research Journal, 18: 15-25. 